\title{
Ipragliflozin-induced adipose expansion inhibits cuff-induced vascular remodeling in mice
}

Kentaro Mori ${ }^{1}$, Kyoichiro Tsuchiya ${ }^{1 *}$, Suguru Nakamura' ${ }^{1}$, Yasutaka Miyachi ${ }^{2}$, Kumiko Shiba ${ }^{2}$, Yoshihiro Ogawa ${ }^{3,4,5}$ and Kenichiro Kitamura ${ }^{1}$

\begin{abstract}
Background: Perivascular adipose tissue (PVAT) plays a critical role in the pathogenesis of cardiovascular disease. It is unclear whether inhibition of sodium glucose cotransporter 2 (SGLT2) in subjects with type 2 diabetes (T2DM) could affect PVAT characters, and whether the SGLT2 inhibitors-induced changes of adipose tissue, especially the alternation of adipose tissue-derived secretory factors, affect vascular pathophysiology.

Methods: Western-type diet (WD) fed wild-type mice were treated with or without an SGLT2 inhibitor ipragliflozin (Ipra) for 10 weeks. WEHI 274.1 and primary vascular smooth muscle cells were incubated with conditioned media (CM) of epididymal adipose tissue (Epi) or abdominal PVAT of Ipra- or vehicle-treated mice fed a WD. Epi of Ipra- or vehicle-treated mice fed a WD was implanted onto cuff-placed femoral arteries of apoE-deficient mice.

Results: Ipra increased adipocyte size associated with decreased expression of pro-inflammatory and fibrosis-related genes in abdominal PVAT of WD-fed mice. Ipra also suppressed WD-induced macrophages accumulation, fibrosis, and adipocyte death in abdominal PVAT. In CM of abdominal PVAT from Ipra-treated mice, concentration of leptin was significantly lower than that from vehicle-treated mice. In vitro, migration of WEHI 274.1 and primary vascular smooth muscle cells were more enhanced by CM of Epi or abdominal PVAT from vehicle-treated mice than that from Ipratreated mice. Perivascular implantation of Epi from Ipra-treated mice to apolipoprotein E-deficient mice attenuated cuff-induced neointimal hyperplasia and vascular remodeling compared to that from vehicle-treated mice.

Conclusions: The Ipra-induced changes of abdominal PVAT will lead to a better understanding of unveiled mechanisms by which SGLT2 inhibitors prevent cardiovascular complications in T2DM, and the development of new therapeutic strategies targeting PVAT.
\end{abstract}

Keywords: Vascular remodeling, Perivascular adipose tissue, Healthy adipose expansion, Cuff injury

\section{Background}

Sodium glucose cotransporter 2 (SGLT2) inhibitors are a class of oral hypoglycemic agents that work by decreasing glucose reabsorption in the renal proximal tubules to promote urinary glucose excretion. The net effect of increased renal glucose excretion has dual effects of insulin-independent glycemic control and caloric loss,

\footnotetext{
*Correspondence: tsuchiyak@yamanashi.ac.jp

${ }^{1}$ Third Department of Internal Medicine, Interdisciplinary Graduate School of Medicine and Engineering, University of Yamanashi, 1110 Shimokato, Chuo, Yamanashi 4093898, Japan

Full list of author information is available at the end of the article
}

thereby leading to improve insulin sensitivity. Accumulating evidence suggests that SGLT2 inhibitors provide multiple benefits to reduce major cardiovascular adverse events including myocardial infarction, stroke, and heart failure in patients with type 2 diabetes (T2DM) $[1,2]$. Recent clinical [3, 4] and experimental [5] evidence have further confirmed that SGLT2 inhibitors have benefits on atherosclerotic cardiovascular events. Although both hemodynamic and metabolic explanations have been proposed, the mechanisms underlying the protective effects of SGLT2 inhibitors on cardiovascular complications among T2DM still remain to be explored. 
Perivascular adipose tissue (PVAT) surrounds vasculature and has been suggested to play an important role in the pathogenesis of cardiovascular disease [6]. PVAT not only stores triglycerides and functions as structural support for vessels, but also secretes a wide variety of biologically active molecules to control vascular function as well as remodeling. In obesity or T2DM, PVAT dominantly secrete pro-inflammatory and pro-atherogenic cytokines, which could cause local endothelial dysfunction, thus contributing to the progression of systemic and local vascular diseases. For instance, leptin, visfatin, and monocyte chemoattractant protein-1 (MCP-1), which modulate vascular tone, macrophages recruitment and activation, vascular smooth muscle cells (VSMCs) migration and proliferation [7], are secreted from PVAT to potentially promote neointimal hyperplasia and formation in adjacent blood vessel by the paracrine fashion [8]. Among antidiabetic agents, alogliptin reported to improve endothelial function by promoting autophagy in perivascular adipose tissue of obese mice through a glucagon-like peptide-1-dependent mechanism [9]. As human evidence, secreted frizzled-related protein 4 derived from epicardial adipose tissue, which can be recognized as PVAT of coronary arteries, is reported to increase in patients with coronary artery disease [10]. It therefore suggests that PVAT has been considered as a potential therapeutic target for treatment of atherosclerosis associated with obesity and/or diabetes [11].

Others and we recently reported that the SGLT2 inhibitors promotes fat accumulation in epididymal adipose tissue (Epi) of diet-induced obese mice without deteriorating adipose inflammation and/or fibrosis, which may be referred to as "healthy adipose expansion" [12-16]. However, it remains unknown whether SGLT2 inhibitors also alter characters of PVAT to the "healthy adipose expansion", and if so, whether the SGLT2 inhibitorsinduced changes of adipose tissue, especially the alternation of adipose tissue-derived secretory factors, affect vascular pathophysiology.

Here we demonstrated that treatment with Ipra could increase adipocyte size with decreased expression of proinflammatory and pro-fibrotic genes in abdominal PVAT of Western-type diet (WD)-fed wild-type (WT) mice. Ipra also suppressed WD-induced macrophages accumulation and adipocyte death in abdominal PVAT, and perivascular implantation of Epi from Ipra-treated mice to apoE-deficient mice attenuated cuff-induced neointimal hyperplasia and vascular remodeling. This will lead to a better understanding of unveiled mechanisms by which SGLT2 inhibitors prevent cardiovascular complications in T2DM, and the development of new therapeutic strategies focusing on PVAT.

\section{Materials and methods}

\section{Animals and experimental protocol}

Male C57BL/6 J WT mice were purchased from CLEA Japan, Inc. (Tokyo, Japan) and Charles River Laboratories Japan, Inc. (Kanagawa, Japan). Male apolipoprotein-E (ApoE)-knockout mice were purchased from Charles River Laboratories Japan, Inc. The animals were allowed free access to water and a standard diet (SD, CE-2; $343 \mathrm{kcal} / 100 \mathrm{~g}, 12.6 \%$ energy as fat; CLEA Japan, Inc.). Ipragliflozin (Ipra: provided by Astellas Pharma Inc., Tokyo, Japan) was dissolved in dimethyl sulfoxide (DMSO; Nacalai Tesque, Inc., Kyoto, Japan) at $0.04 \%$ (v/v) and added into the drinking water. In a Western type diet (WD) feeding experiments, 8-week-old WT mice were fed a WD (D12079B, 41\% fat, 43\% carbohydrate, and 17\% protein content on an energy basis, Research Diets Inc., New Brunswick, NJ, USA) for 8 weeks, and thereafter a WD with the vehicle or Ipra for 10 weeks. Age matched control male WT mice were fed a SD throughout the experiment period. Body weight and blood glucose were measured every week. Thoracic PVAT from distal end of the aortic arch to the diaphragm, and abdominal PVAT from diaphragm to bifurcation were harvested. Upper and lower half of the PVATs were subjected to histological and mRNA/protein analyses, respectively. Concentration of Ipra in the drinking water was changed every week based on daily water consumption and body weight to adjust $10 \mathrm{mg} / \mathrm{kg} /$ day. At the end of the experiment, the animals were sacrificed under intraperitoneal pentobarbital anesthesia $(30 \mathrm{mg} / \mathrm{kg})$ after $16 \mathrm{~h}$ of fasting.

\section{Biochemical assays}

Blood glucose was measured using a glucometer (OneTouch Verio ${ }^{\circledR}$ IQ; LifeScan Japan Co., Ltd., Tokyo, Japan). Serum total cholesterol, non-esterified fatty acid, and triglyceride (TG) concentrations were determined with Wako Cholesterol E, NEFA C-Test Wako, and TG E-Test Wako (Wako Pure Chemical Industries, Ltd., Osaka, Japan), respectively. Insulin (Morinaga Institute of Biological Science, Inc., Kanagawa, Japan), leptin (FUJIFILM Wako Shibayagi Co., Gunma, Japan), high mobility group box 1 (HMGB1) (FUJIFILM Wako Shibayagi Co.), and fatty acid binding protein 4 (FABP4) (MyBioSource, Inc., CA, USA) concentrations were measured with an enzyme-linked immune sorbent assay kits.

\section{Histological analysis}

The aorta and PVAT were fixed with $4 \%$ paraformaldehyde and embedded in paraffin and prepared as slides (4- $\mu \mathrm{m}$-thick sections). PVAT sections were stained with hematoxylin and eosin (HE). For the measurement of adipocyte cell size, $>250$ cells were counted per each section using an image analysis software (NIH Image J software). 
Macrophages in the PVAT were immunohistochemically detected using a rat monoclonal F4/80 antibody (MCA497GA; Abd Serotec, Kidlington, UK). Neointima formation in cuff-injured femoral arteries were stained as immunohistochemically detected using a $\alpha$-smooth muscle actin ( $\alpha$-SMA) antibody (ab5694, Abcam, Cambridge, UK). Elastica-van Gieson and Masson trichrome staining were also performed in femoral arteries. Binding of primary antibody was visualized using $\mathrm{DAB}+$ chromogen (Dako, Glostrup, Denmark). PVAT was stained using Sirius red for the assessment of fibrosis; positive areas were measured using NIH Image J software. TUNEL staining was performed using the ApopTag Plus Peroxidase In Situ Apoptosis Detection Kit (Millipore, Billerica, MA, USA) according to the manufacturer's instruction. TUNEL-positive cells were counted in the whole area of the section. All microscopic images were acquired using the Keyence BZ-9000 microscope.

\section{Quantitative RT-PCR}

Total RNA of the PVAT was isolated using Sepasol reagent (Nacalai Tesque, Inc.). RNA was reverse transcribed with Random Primer (Thermo Fisher Scientific Inc., Waltham, MA, USA) and ReverTra Ace (Toyobo Co., Ltd., Osaka, Japan). Quantitative RT-PCR was performed using StepOnePlus Real-time PCR System with Fast SYBR Green Master Mix Reagent (Thermo Fisher Scientific Inc.). Primers are listed in S Table. Data were normalized to the $36 \mathrm{~b} 4$ levels, and analyzed by the comparative CT method.

\section{Western blotting}

PVAT was homogenized in a lysis buffer (2\% SDS, $4 \mathrm{M}$ Urea, $1 \mathrm{mM}$ EDTA, $150 \mathrm{mM} \mathrm{NaCl}, 50 \mathrm{mM}$ Tris $\mathrm{pH}$ 8.0). Immunoblotting was performed with a phospho (Ser473)-Akt (9271, Cell Signaling Technology, Danvers, MA, USA), a total Akt antibody (9272, Cell Signaling Technology), HMGB1 (6893, Cell Signaling Technology), $\beta$-actin (4970, Cell Signaling Technology). Immunoblots were detected and analyzed with ECL Prime Western Blotting Detection Reagent and ImageQuant LAS 4000 mini (GE Healthcare, Little Chalfont, UK).

\section{Conditioned media collection from adipose tissue}

Experimental mice ( $\mathrm{n}=2$ in each group) were sacrificed by intraperitoneal pentobarbital anesthesia. The epididymal adipose tissue (Epi) or abdominal PVAT from diaphragm to bifurcation were dissected in DMEM with $1 \%$ penicillin/streptomycin (P/S), $0.25 \%$ BSA. Tissue pieces from each mice were weighed as $100 \mathrm{mg}$ (Epi) or $20 \mathrm{mg}$ (abdominal PVAT), then minced 20 times, and incubated with $1 \mathrm{ml}$ of DMEM with $1 \% \mathrm{P} / \mathrm{S}, 0.25 \% \mathrm{BSA}$ in 24-well culture plates (Costar, Corning, NY, USA) at $37 \mathrm{C}$ for $5 \mathrm{~h}$.

\section{Chemotaxis assays}

The effects of CM of Epi on monocytes recruitment were determined using Boyden chambers (Cell Biolabs, CytoSelect ${ }^{\mathrm{TM}}$ 24-Well Cell Migration Assay, $5 \mu \mathrm{m}$ ). Murine monocytes (WEHI 274.1 cells, purchased from ATCC) were initially cultured in DMEM with $10 \%$ FBS and $1 \% \mathrm{P} / \mathrm{S}$, and split twice a week to obtain a sufficient cell number. For experiments, WEHI 274.1 cells were plated into the upper chamber at 100,000 cells per $100 \mu \mathrm{l}$ DMEM with $1 \% \mathrm{P} / \mathrm{S}$, while $\mathrm{CM}$ diluted twice by DMEM was filled into the lower one with or without anti-MCP-1 blocking antibody (R\&D Systems, Inc, $\mathrm{MN}$, USA). After $12 \mathrm{~h}$, the number of migrated cells was counted on dissected membranes using Nikon Eclipse Ti microscope.

\section{Scratch test}

Vascular smooth muscle cells were isolated and cultured from 4-week-old WT male as previously described [17]. Primary VSMCs were plated in 12-well plates at a concentration of 500,000 cells per $2 \mathrm{ml}$ of DMEM with $20 \%$ FBS and $1 \% \mathrm{P} / \mathrm{S}$. The media was changed to DMEM with $0.25 \%$ BSA when an adherent monolayer was obtained. Scratch was created by a $100 \mu$ pipette tip in the monolayer and the cells were washed 3 times with DMEM with $0.25 \%$ BSA. The cells were then stimulated with rat recombinant platelet-derived growth factor (PDGF)-BB (R\&D Systems) in CM diluted twice by DMEM with or without LY294002 (Cell Signaling Technology). After $24 \mathrm{~h}$, images of the scratch wounds were taken and measured by Image-J software.

\section{Perivascular implantation of adipose tissue and cuff injury}

Surgery was carried out on 8-week-old male mice as described previously [18] with some modifications. In brief, the femoral artery was isolated from surrounding tissues under anesthesia, and then a polyethylene tube (PE-20; $\mathrm{BD})$ was loosely placed around the artery. Fifty $\mathrm{mg}$ of Epi was taken from WD-fed or WD/Iprafed mouse, followed by placed onto the artery after cuff placement. The mice were sacrificed 4 weeks after surgery, and the implanted Epi and arteries were obtained for analyses.

\section{Statistical analysis}

For normally distributed values, data were expressed as mean \pm standard error of the mean (SEM), and were compared using student's $t$ test, or analysis of variance 
(ANOVA) with post hoc testing. For non-Gaussian distributed values, data were expressed as box-and-whisker plots with median values and $10,25,75$, and $90 \%$. Nonparametric statistical analysis was performed with the Mann-Whitney or Kruskal-Wallis test with Dunn post hoc test. $p<0.05$ was considered to be statistically significant. Statistical analysis was performed using Prism 7 (GraphPad software, Inc., CA, USA).

\section{Results}

Ipra increases adipocyte size with enhanced insulin signaling in abdominal PVAT of WD-fed mice

First we put WT mice on WD for 10 weeks with or without Ipra treatment. Ipra did not affect the changes of body weight during the WD feeding as previously shown in mice fed a HFD [19] (Fig. 1a). In contrast, Ipra treatment significantly increased Epi weight to body weight ratio (Additional file 1: Fig. S1a). Ipra significantly attenuated WD-induced hyperglycemia (Fig. 1b, c) and insulin resistance with a trend of decrease of serum insulin concentration (Fig. 1d, e). Ipra did not change serum lipid profile of WD-fed mice (Additional file 1: Fig. S1b). Histological analysis revealed that Ipra treatment increased adipocyte size in abdominal PVAT of WD-fed mice (Fig. 1f-h), accompanied with increased expression of lipid storage marker perilipin-1 (Fig. 1i). Ipra did not affect adipocyte size in thoracic PVAT (Additional file 1: Fig. S1c). Whereas phosphorylation of Akt (Ser473) in abdominal PVAT of WD-fed mice was significantly suppressed compared to SD-fed mice, its suppression was not observed in Ipra-treated mice (Fig. 1j).

\section{Ipra attenuates inflammation, fibrosis, and adipocyte death in abdominal PVAT of WD-fed mice}

In abdominal PVAT, inflammation ( $\mathrm{Ccl} 2, \mathrm{Ccr} 2$, and Emr1)- and fibrosis (Col1a1, Col1a2, and Fn1)-related genes were upregulated in WD-fed mice compared to SD-fed mice, which were significantly or tended to be inhibited by Ipra treatment (Fig. 2a). Expression of $I l 1 b$, Il6, and Tnf were unaffected by WD feeding and Ipra treatment (Fig. 2a). Expression of Ccl2, Ccr2, and Emr1 in thoracic PVAT was not changed by Ipra treatment (Additional file 1: Fig. S1d). Accordingly, immunostaining for a macrophage marker F4/80 revealed that Ipra treatment effectively suppressed macrophage infiltration and crown-like structure (CLS) formation in abdominal PVAT of WD-fed mice (Fig. 2b). Fibrosis in abdominal PVAT assessed by Sirius red staining, which is reported to tightly associated with tissue inflammation characterized by macrophage infiltration [20], was also significantly reduced in Ipra-treated mice (Fig. 2c).

It has been reported that the number of macrophages in obese adipose tissue positively correlates adipocyte apoptosis [21]; in consistent with reduced CLS in abdominal PVAT of Ipra-treated mice, Ipra significantly decreased the number of TUNEL-positive cells in WDfed mice as compared to vehicle-treated mice (Fig. 2d). Dying adipocytes in obese adipose tissue may contribute to the recruitment of immune cells or release of intracellular molecules of adipocytes known as damage-associated molecular patterns [22]. A nuclear protein, high mobility group box 1 (HMGB1), is one of the damageassociated molecular patterns and released from dying adipocytes to potentially promote atherosclerosis and vascular remodeling $[23,24]$. As expected, Ipra tended to reduce protein expression of HMGB1 in abdominal PVAT (Fig. 2e), and suppressed HMGB1 release from isolated Epi of WD-fed mice into CM (data not shown).

\section{Ipra-induced changes of secretory factors from Epi or abdominal PVAT inhibit monocytes and VSMCs migration in vitro}

We further examined whether changes of secretory factors from adipose tissue of Ipra-treated mice affect functions of macrophages and VSMCs related to atherosclerosis and vascular remodeling. An in vitro chemotaxis assay revealed that $\mathrm{CM}$ of Epi from vehicle-treated mice fed a WD significantly enhanced monocyte migration as compared to that from SD-fed mice, whose effect was attenuated in CM of Epi from Ipra-treated mice (Fig. 3a). Pretreatment with a neutralizing anti-MCP-1 antibody also inhibited the increase of monocyte migration by $\mathrm{CM}$ of Epi from vehicle-treated mice, and it also diminished the difference of monocyte migration stimulated by $\mathrm{CM}$ of Epi from Ipra- and vehicle-treated mice (Fig. 3a).

Leptin enhances VSMCs proliferation, migration, and neointimal hyperplasia via a PI3 K-dependent fashion [25]. In abdominal PVAT of Ipra-treated mice, including Lep, expression of Angptl2 [26], Pdgfb [27], and Nampt [28], whose products have been shown to promote VSMCs proliferation, migration, and/or neointimal hyperplasia, were significantly suppressed compared to that of vehicle-treated mice (Fig. 3b). Leptin concentration in CM of abdominal PVAT from Ipra-treated mice was also significantly lower than that from vehicle-treated mice (Fig. 3c). Whereas the CM of abdominal PVAT from vehicle-treated mice enhanced platelet-derived growth factor (PDGF)-BB-induced VSMCs migration in vitro, its effect was significantly attenuated in $\mathrm{CM}$ of abdominal PVAT from Ipra-treated mice (Fig. 3d). Furthermore, pretreatment with LY294002, a PI3K inhibitor, also inhibited VSMCs migration by the CM of abdominal PVAT from vehicle-treated mice (Fig. 3d). Concentration of fatty acid binding protein 4 (FABP4), which is also reported to enhance VSMCs proliferation and migration [29], and inflammatory responses in macrophages [30], 

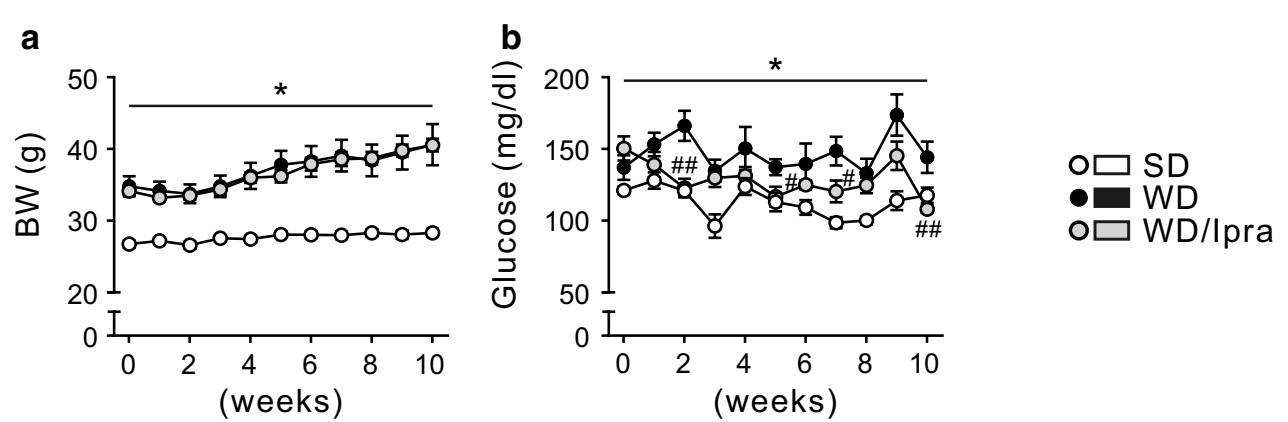

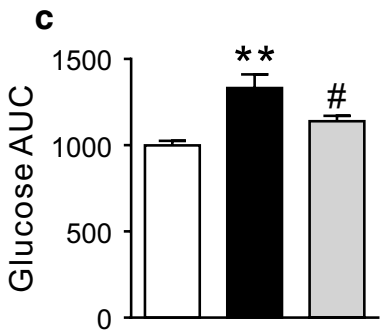

d

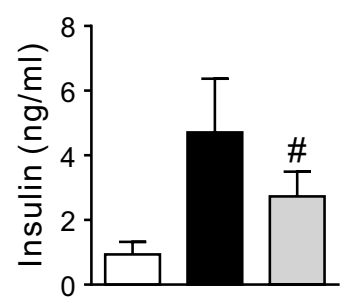

f

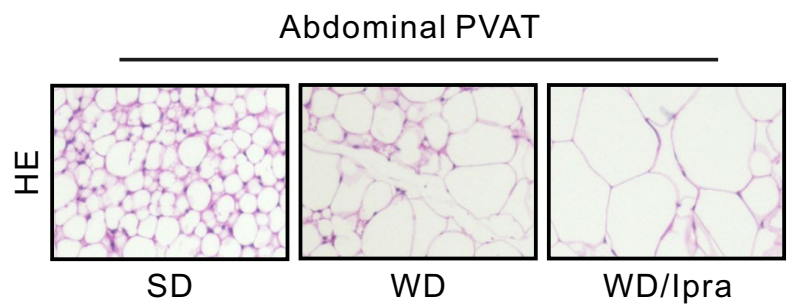

h

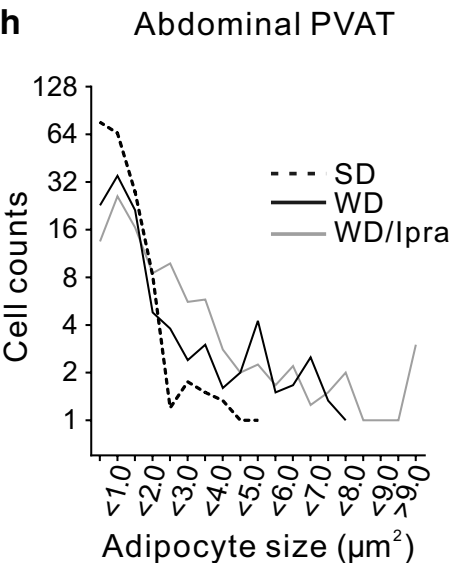

i

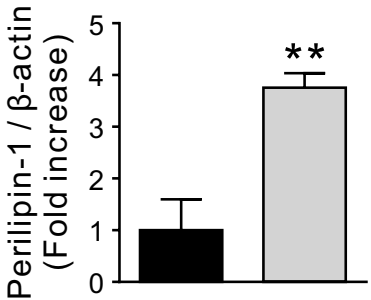

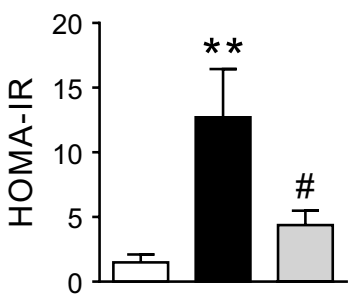

g Abdominal PVAT

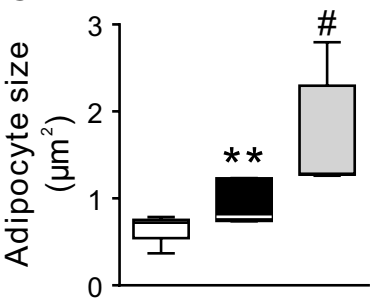

j Abdominal

PVAT

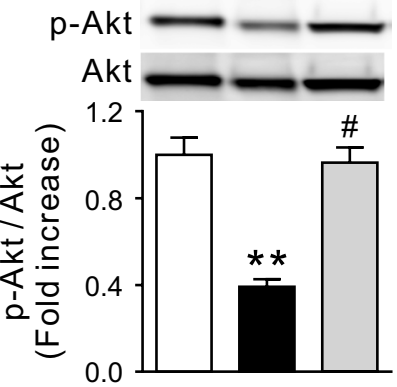

Fig. 1 Ipra increases adipocyte size with enhanced insulin signaling in abdominal PVAT of WD-fed mice. The changes in $\mathbf{a}$ body weight and $\mathbf{b}$ blood glucose, and $\mathbf{c}$ area under curve (AUC) of blood glucose in SD- or WD-fed WT mice during Ipra treatment for 10 weeks. $\mathbf{d}$ Plasma insulin concentration and $\mathbf{e}$ HOMA-IR after 10 weeks of Ipra treatment. $\mathbf{f}$ Hematoxylin and eosin (HE) staining, and $\mathbf{g}$ quantification and $\mathbf{h}$ histogram of adipocyte size in abdominal PVAT. Quantitative data of $\mathbf{i}$ perilipin-1 and $\mathbf{j}$ phosphorylated Akt (p-Akt) in abdominal PVAT. WT wild-type, SD standard diet, WD Western-type diet, Ipra ipragliflozin, PVAT perivascular adipose tissue. Original magnification, $\times 200 .{ }^{*} p<0.05,{ }^{* *} p<0.01$ vs SD. ${ }^{*} p<0.05$, $\#^{\#}<0.01$ vs WD. $n=6-8$ 



Abdominal PVAT
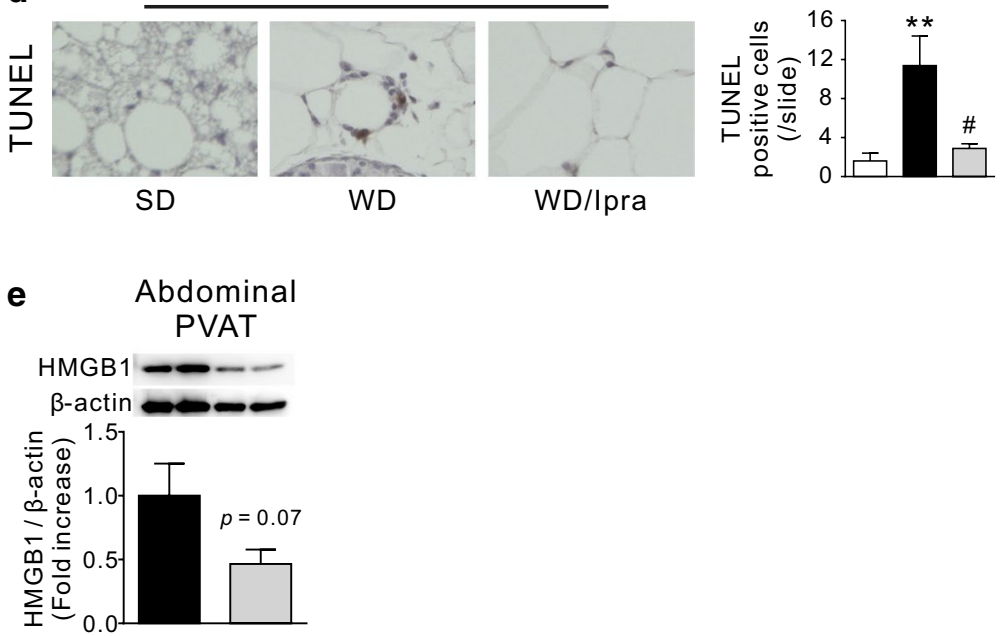

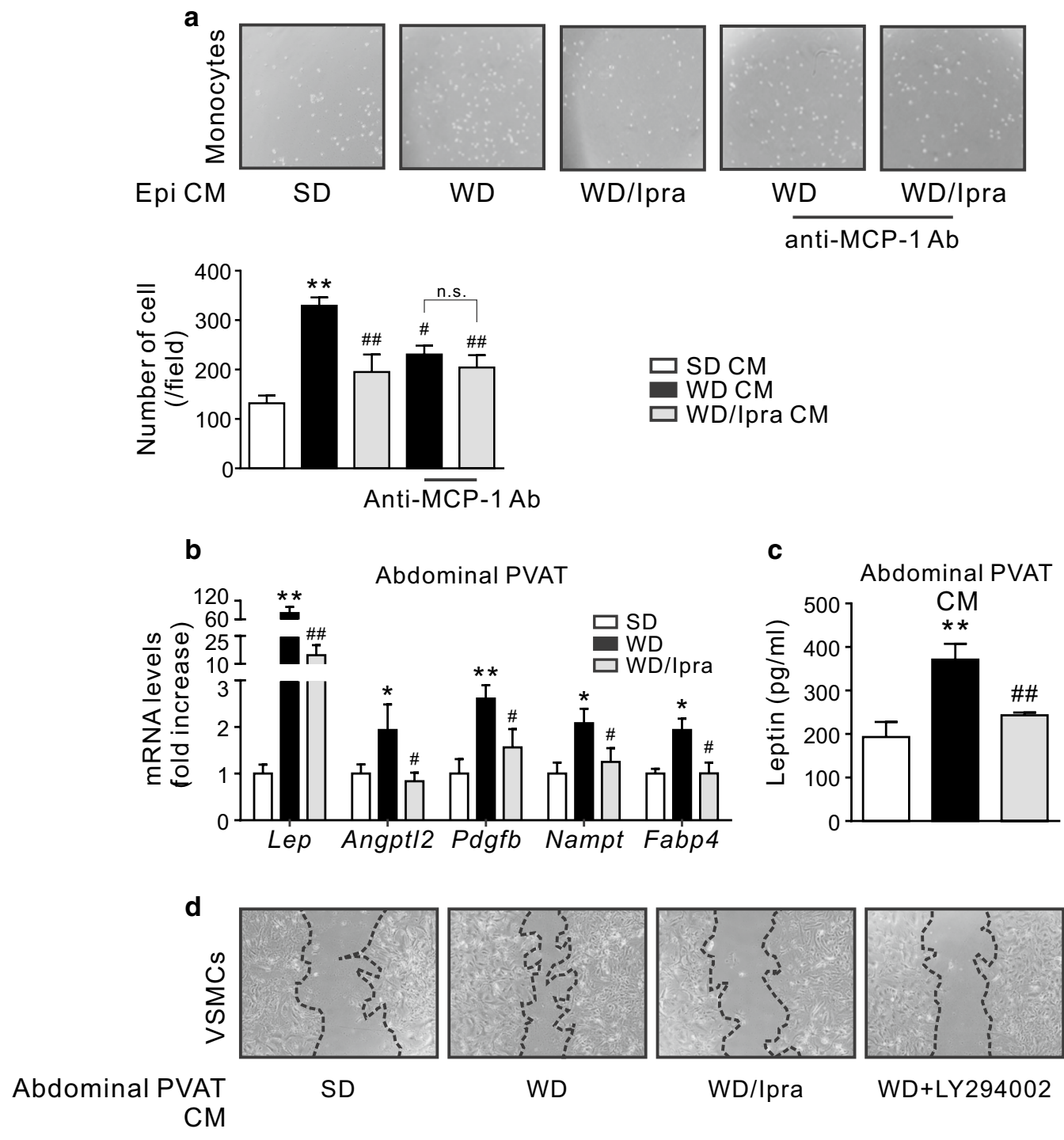

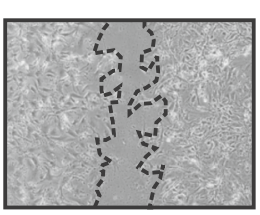

WD

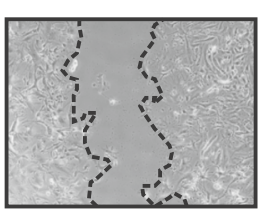

WD/Ipra

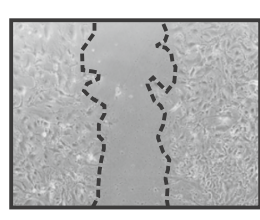

WD+LY294002



Fig. 3 Ipra suppresses monocytes and VSMCS migration. a Representative pictures and quantitative data of migrated monocytes (WEHI274.1 cells) under CM of Epi with or without anti-MCP-1 blocking antibody (anti-MCP-1 Ab, $2 \mu \mathrm{g} / \mathrm{ml}$ ). $\mathbf{b}$ Expression levels of proliferation- and migration-related genes in abdominal PVAT. $\mathbf{c}$ Leptin concentration in CM of abdominal PVAT. $\mathbf{d}$ Representative pictures and quantitative data of PDGF-BB-induced primary cultured VSMCs migration under CM of abdominal PVAT with or without LY294002 (10 $\mu$ M). VSMCs, vascular smooth muscle cells. ns not significant. ${ }^{*} p<0.05,{ }^{* *} p<0.01$ vs SD. ${ }^{\#} p<0.05,{ }^{\# \#} p<0.01$ vs WD. $n=6-8$ 
was significantly lower in CM of Epi from Ipra-treated mice, and showed trend of decrease in CM of abdominal PVAT from Ipra-treated mice (Additional file 1: Fig. S2a). Plasma concentration of FABP4 was lower in Ipra-treated mice compared to that of vehicle-treated mice (Additional file 1: Fig. S2b).
Perivascular implantation of adipose tissue

from Ipra-treated mice suppresses cuff-induced neointimal hyperplasia and vascular remodeling in ApoE-knockout mice

We finally asked whether the changes of characters in adipose tissue by Ipra affects neointimal hyperplasia and vascular remodeling in vivo, using the femoral

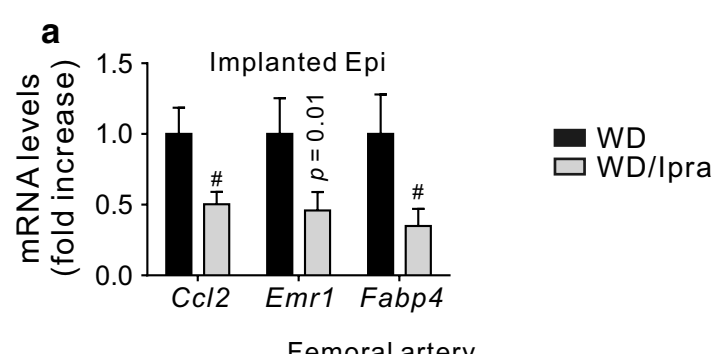

b

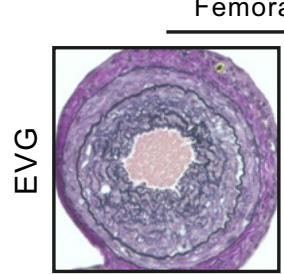

Implanted Epi WD

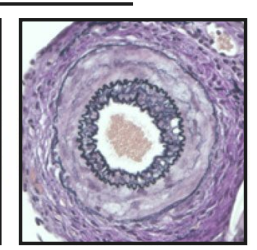

WD/Ipra


WD/Ipra



Implanted Epi WD

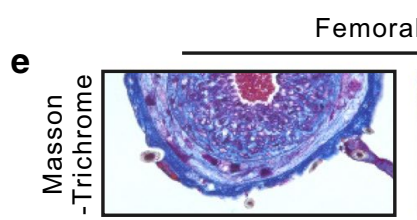

Implanted Epi

WD



WD/Ipra

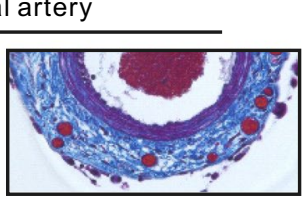

WD/Ipra
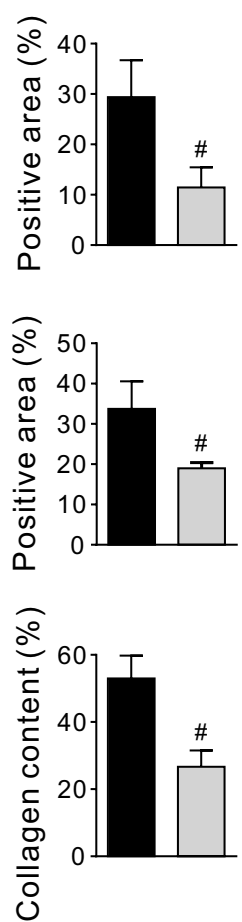

Fig. 4 Perivascular implantation of adipose tissue from Ipra-treated mice suppresses cuff-induced neointimal hyperplasia and vascular remodeling in ApoE-knockout mice. a Gene expression of CCl2, Emr1 and Fabp4 in implanted Epi after 4 weeks of implantation. b Representative pictures of EVG staining, and quantitative data of intima and media area in femoral arteries of ApoE-knockout mice 4 weeks after surgery. c F $4 / 80$ and $\mathbf{d}$ a-SMA immunostaining, and e Masson-Trichrome staining. EVG Elastica-van Gieson, SMA smooth muscle actin. Original magnification, $\times 200 .{ }^{\#} p<0.05$ vs WD. $n=7$ 
artery cuff model; Epi from vehicle- or Ipra-treated mice fed a WD were implanted over a cuff placed around a femoral artery of ApoE-knockout mice. Four weeks after surgery, viable implanted Epi with vascularization were confirmed (Additional file 1: Fig. S3). Downregulation of Ccl2, Emr1, and Fabp4 in implanted Epi from Ipra-treated mice remained 4 weeks after surgery (Fig. 4a). Neointimal hyperplasia assessed by intima area and intima to media ratio were significantly attenuated in ApoE-knockout mice implanted with Epi from Ipra-treated mice compared to vehicle-treated mice (Fig. 4b). The reduced neointimal hyperplasia accompanied with suppressed macrophage infiltration, VSMCs proliferation, and fibrosis (Fig. 4c-e).

\section{Discussion}

The present study demonstrated that Ipra increased adipocyte size in abdominal PVAT in WD-induced obese and diabetic mice, which is consistent with our previous observation in Epi of diet-induced obese mice treated with SGLT2 inhibitors [19, 31, 32]. The adipocyte hypertrophy in abdominal PVAT accompanied a decrease of inflammation and fibrosis, which corresponded to "healthy adipose expansion" [33]. In addition to multiple metabolic benefits by SGLT2 inhibitors such as improvement of hyperglycemia, obesity, and dyslipidemia that could influence cardiovascular mortality, the present study proposed novel mechanisms by which SGLT2 inhibitors prevented vascular complications in T2DM via modulating PVAT characters.

\section{Possible mechanisms of the "healthy adipose expansion" in abdominal PVAT}

We previously observed same phenotypes of the adipose expansion in Epi of Ipra-treated HFD-fed mice [13]; increased adipocyte size and reduced number of CLS were observed in Epi of Ipra-treated mice fed a HFD. It suggests that Ipra increased the lipid-storage capacity of adipocytes and inhibited adipocyte death followed by macrophage accumulation. These results are consistent with a study using adipocyte-specific inducible phosphatase and tensin homologue (PTEN)-knockout mice, which exhibit enhanced insulin signaling in adipocytes. Whereas PTEN-knockout mice gained more adipose tissue during HFD feeding, they showed enhanced insulin sensitivity, improved hepatic steatosis, and reduced adipose tissue inflammation [34]. Then, improvement of insulin resistance in adipocytes could cause the adipose expansion without deteriorating inflammation, and might lead to attenuation of ectopic lipid accumulation, which can be called as "healthy adipose tissue expansion".

As a common action of PTEN-knockout mice, Ipra treatment improved insulin resistance via its insulin-independent glucose-lowering action; indeed, the present study has shown that Ipra treatment improves both hyperglycemia and hyperinsulinemia with increased Akt phosphorylation in abdominal PVAT compared to vehicle treatment. Although phosphorylation of Akt is not a specific marker of insulin signaling, improved hyperglycemia, hyperinsulinemia, and systemic insulin resistance assessed by HOMA-R indirectly suggest that increased phosphorylation of Akt is presumed to reflect improved insulin sensitivity in abdominal PVAT of Ipratreated mice. Furthermore, Ipra treatment significantly increased Epi weight to body weight ratio despite of comparable whole body weight to vehicle-treated mice, suggesting the "healthy adipose tissue expansion". Taken together, these observation suggest that enhanced insulin signaling in abdominal PVAT by Ipra was likely to play an main role in promoting the "healthy adipose expansion". The detailed molecular mechanisms by which enhancement of insulin signaling in adipocytes causes the "healthy adipose expansion" require further studies.

\section{Cell death in PVAT and vascular injury}

In addition to reduced expression of inflammationrelated genes, increased adipocyte size, and reduced number of CLS and TUNEL-positive cells were observed in abdominal PVAT of Ipra-treated mice. It suggests that Ipra increases the lipid-storage capacity of adipocytes and inhibited adipocyte death followed by macrophage accumulation as we previously reported in Epi [19, 32]. Indeed, expression of HMGB1 protein, which is passively released by injured or dying cells and aggravates inflammatory processes in various cells [35], was markedly reduced in abdominal PVAT of Ipra-treated mice compared to that of vehicle-treated mice. HMGB1 is known as not only a hallmark of cell death, but also a cytokine; in blood vessels, high levels of extracellular HMGB1 have been detected in human atherosclerotic plaque [36], and are reportedly implicated in vascular inflammation by potentiating inflammatory responses including macrophage migration and activation [23, 24]. In VSMCs, it has been shown that HMGB1 modulates their phenotype toward the activated synthetic phenotype and stimulates MCP-1 gene expression through toll-like receptor 4 [24, 37]. Taken together, as well as the altered gene expression in abdominal PVAT, decreased adipocyte death in abdominal PVAT by Ipra is also considered to inhibit the development of vascular dysfunction and remodeling, especially in the setting of obesity as in the present study.

\section{Possible mechanisms of suppressed cuff-induced vascular remodeling}

In the present study, perivascular implantation of Epi from Ipra-treated mice prevented cuff-induced 
neointimal hyperplasia and vascular remodeling in femoral arteries of ApoE-knockout mice compared to that from vehicle-treated mice. It suggests that Ipra-induced changes of secretory factors from Epi of WD-fed mice may attenuate these histological changes in the arteries. We implanted Epi instead of PVAT due to technical difficulties, however, it is supposed that PVAT-derived secretory factors would also affect cuff-induced vascular remodeling as paracrine manner. Although dissecting the responsible secretory factors changed by Ipra is beyond the present study, we assumed that, considered from ex vivo and in vitro studies, decreased HMGB1, leptin, MCP-1, NAMPT, PDGF-B and/or FABP4 expression/ production from implanted adipose tissue could play inhibitory roles for the development of neointimal hyperplasia and vascular remodeling via attenuating monocyte migration and VSMCs proliferation. HMGB1 and MCP-1 reportedly play roles mainly in inflammatory cells such as macrophages, neutrophils, and lymphocytes to recruit them into the vascular adventitia. It has been shown that neutralization of HMGB1 reduces development of atherosclerosis in apolipoprotein E-deficient mice [38], suggesting significant contribution of decreased HMGB1 by Ipra to the inhibition of vascular remodeling. In contrast, leptin, PDGF-B, and visfatin, although they are also reported to affect inflammatory cells [39, 40], can affect VSMCs to enhance phenotype switch to proliferative character, and promote migration to the neointima [6, $39,41]$. In addition, FABP4 has been reported to induce proliferation and migration of vascular smooth muscle cells through a MAPK-dependent pathway [29]. In macrophages, treatment with recombinant FABP4 significantly increased gene expression of inflammatory markers in a dose-dependent manner [42]. FABP4 is also reported to ectopically express in endothelial cells to promote neointima formation by wire-induced vascular injury [43]. Then, decreased gene and/or protein expression of these factors derived from abdominal PVAT are presumed to coordinately and complementarily affect vascular cells, leading to inhibition of cuff-induced vascular remodeling.

\section{Persistent of SGLT2 inhibitors-induced changes in implanted adipose tissues}

Moreover, it is noteworthy that expression pattern of pro-inflammatory genes in implanted adipose tissues partly remained similar to that before implantation even 4 weeks after implantation. It suggests some cellautonomous mechanisms that maintain altered gene expression, referred to as "cellular memory", after exposure to SGLT2 inhibitors, or SGLT2 inhibitors-induced changes of extracellular factors. As one of the possible mechanism to maintain the "cellular memory", epigenetic modifications are generally speculated; obesity and/or diabetes are shown to associate DNA and histone methylation levels in various genes of adipose tissue [44], including inflammatory genes $[45,46]$. Whereas evidences related to SGLT2i-induced epigenetic changes are limited, a previous paper has shown that treatment with SGLT2i affects gene expression in aortae associated with epigenetic modifications; treatment with empagliflozin for 6 week prevented induction of inflammationand glucotoxicity-related genes in aortae of Zucker fatty rats, along to suppression of activating epigenetic mark trimethylation of histone $\mathrm{H} 3$ at lysine4 (H3K4me3) of these genes [47]. It is therefore possible that Ipra attenuates WD-induced changes of inflammatory genes associated with epigenetic changes in adipose tissue, and the epigenetic changes may contribute to maintain the gene expression levels even after Epi extraction. The precise molecular, genetic, and epigenetic mechanisms by which gene expression are maintained in adipose tissue from Ipra-treated mice needs to be elucidated.

\section{Differences between thoracic and abdominal PVAT}

Unlike abdominal PVAT, Ipra did not affect adipocyte size, and Ccl2, Ccr2, or Emr1 expression in thoracic PVAT. It has been known that the structural and physiological characteristics of PVAT vary according to its location. Abdominal PVAT resembles white adipose tissue (WAT), with less differentiated adipocytes, poor vascularization, a specific profile of cytokines production, and contains infiltrates of macrophages [48]. On the other hand, thoracic PVAT exhibits features that resemble brown adipose tissue (BAT) rather than WAT $[49,50]$. Although precise mechanisms by which Ipra differently affects thoracic and abdominal PVAT remains unclear, the distinct differences between thoracic and abdominal PVAT in response to Ipra may attribute these basic cellular characteristics. Furthermore, a previous report has suggested that insulin-induced Akt phosphorylation is more pronounced in WAT than BAT in mice [51], suggesting that WAT appears to be more insulinsensitive than BAT. Assuming that insulin sensitivity is crucial for promoting the healthy adipose expansion, the difference of insulin sensitivity may result in the differences of characters between thoracic and abdominal PVAT.

\section{Conclusions}

The present study proposes a novel mechanism by which a SGLT2 inhibitor Ipra induced adipocyte hypertrophy without increasing inflammation, fibrosis, and adipocyte death in abdominal PVAT of WD-fed mice. The data of this study also imply a novel inter-organ network between 
kidney, adipose tissue and vasculature, and thus suggest a clinical implication for the prevention and treatment of obesity and/or T2DM-associated cardiovascular complications through pharmacological intervention.

\section{Additional file}

Additional file 1. Additional figures and tables.

\section{Abbreviations}

PVAT: perivascular adipose tissue; Ipra: ipragliflozin; HMGB1: high mobility group box 1; CM: conditioned media; SGLT2: sodium-glucose cotransporter 2 inhibitor; T2DM: type 2 diabetes mellitus; WAT: white adipose tissue; BAT: brown adipose tissue; MCP-1: monocyte chemoattractant protein-1; FABP-4: fatty acid binding protein 4; VSMCs: vascular smooth muscle cells.

\section{Acknowledgements}

We thank all lab members for helpful discussion.

\section{Authors' contributions}

KM and KT participated in the study design, execution of the studies, data acquisition, data analysis, interpretation and in the writing of the manuscript. SN, YM, and KS participated in data acquisition, data interpretation in the writing and final approval of the manuscript. $\mathrm{YO}$ and KK participated in the study design, data interpretation, and supervision of the project. All authors read and approved the final manuscript.

\section{Funding}

This work was supported by JSPS KAKENHI (Grants-in-Aid for Scientific Research)

\section{Availability of data and materials}

The datasets used and/or analyzed during the current study are available from the corresponding author on reasonable request.

\section{Ethics approval and consent to participate}

This study was carried out in strict accordance with the guidelines for the care and use of laboratory animals of the Tokyo Medical and Dental University, and the University of Yamanashi. The protocol was approved by Committees on Animal Research in Tokyo Medical and Dental University, and University of Yamanashi.

\section{Consent for publication}

Not applicable.

\section{Competing interests}

Ipragliflozin was provided by Astellas Pharma Inc., Tokyo, Japan.

\section{Author details}

${ }^{1}$ Third Department of Internal Medicine, Interdisciplinary Graduate School of Medicine and Engineering, University of Yamanashi, 1110 Shimokato, Chuo, Yamanashi 4093898, Japan. ${ }^{2}$ Department of Molecular Endocrinology and Metabolism, Graduate School of Medical and Dental Sciences, Tokyo Medical and Dental University, Tokyo, Japan. ${ }^{3}$ Department of Medicine and Bioregulatory Science, Graduate School of Medical Sciences, Kyushu University, Fukuoka, Japan. ${ }^{4}$ Department of Molecular and Cellular Metabolism, Graduate School of Medical and Dental Sciences, Tokyo Medical and Dental University, Tokyo, Japan. ${ }^{5}$ Japan Agency for Medical Research and Development, CREST, Tokyo, Japan.

Received: 23 March 2019 Accepted: 13 June 2019

Published online: 24 June 2019

\section{References}

1. Zinman B, Wanner C, Lachin JM, Fitchett D, Bluhmki E, Hantel S, Mattheus M, Devins T, Johansen OE, Woerle HJ, et al. Empagliflozin, cardiovascular outcomes, and mortality in type 2 diabetes. N Engl J Med. 2015:373(22):2117-28.

2. Neal B, Perkovic V, Mahaffey KW, de Zeeuw D, Fulcher G, Erondu N, Shaw W, Law G, Desai M, Matthews DR, et al. Canagliflozin and cardiovascular and renal events in type 2 diabetes. N Engl J Med. 2017;377(7):644-57.

3. Monami M, Dicembrini I, Mannucci E. Effects of SGLT-2 inhibitors on mortality and cardiovascular events: a comprehensive meta-analysis of randomized controlled trials. Acta Diabetol. 2017;54(1):19-36.

4. Zelniker TA, Wiviott SD, Raz I, Im K, Goodrich EL, Bonaca MP, Mosenzon O, Kato ET, Cahn A, Furtado RHM, et al. SGLT2 inhibitors for primary and secondary prevention of cardiovascular and renal outcomes in type 2 diabetes: a systematic review and meta-analysis of cardiovascular outcome trials. Lancet. 2019;393(10166):31-9.

5. Al-Sharea A, Murphy AJ, Huggins LA, Hu Y, Goldberg IJ, Nagareddy PR. SGLT2 inhibition reduces atherosclerosis by enhancing lipoprotein clearance in Ldlr(-/-) type 1 diabetic mice. Atherosclerosis. 2018;271:166-76.

6. Qi XY, Qu SL, Xiong WH, Rom O, Chang L, Jiang ZS. Perivascular adipose tissue (PVAT) in atherosclerosis: a double-edged sword. Cardiovasc Diabetol. 2018;17(1):134.

7. Miao CY, Li ZY. The role of perivascular adipose tissue in vascular smooth muscle cell growth. Br J Pharmacol. 2012;165(3):643-58.

8. Maenhaut N, Van de Voorde J. Regulation of vascular tone by adipocytes. BMC Med. 2011;9:25.

9. Zhu B, Li Y, Mei W, He M, Ding Y, Meng B, Zhao H, Xiang G. Alogliptin improves endothelial function by promoting autophagy in perivascular adipose tissue of obese mice through a GLP-1-dependent mechanism. Vascul Pharmacol. 2019;115:55-63.

10. Ji Q, Zhang J, Du Y, Zhu E, Wang Z, Que B, Miao H, Shi S, Qin X, Zhao Y, et al. Human epicardial adipose tissue-derived and circulating secreted frizzled-related protein 4 (SFRP4) levels are increased in patients with coronary artery disease. Cardiovasc Diabetol. 2017;16(1):133.

11. Vargas G, Balcazar-Hernandez LJ, Melgar V, Magrina-Mercado RM, Gonzalez B, Baquera J, Mercado M. An FSH and TSH pituitary adenoma, presenting with precocious puberty and central hyperthyroidism. Endocrinol Diabetes Metab Case Rep. 2017;2017:1.

12. Rutkowski JM, Stern JH, Scherer PE. The cell biology of fat expansion. J Cell Biol. 2015;208(5):501-12.

13. Komiya C, Tsuchiya K, Shiba K, Miyachi Y, Furuke S, Shimazu N, Yamaguchi S, Kanno K, Ogawa Y. Ipragliflozin improves hepatic steatosis in obese mice and liver dysfunction in type 2 diabetic patients irrespective of body weight reduction. PLOS ONE. 2016;11(3):e0151511.

14. Dalmas E, Toubal A, Alzaid F, Blazek K, Eames HL, Lebozec K, Pini M, Hainault I, Montastier E, Denis RG, et al. Irf5 deficiency in macrophages promotes beneficial adipose tissue expansion and insulin sensitivity during obesity. Nat Med. 2015;21(6):610-8.

15. Khan T, Muise ES, lyengar P, Wang ZV, Chandalia M, Abate N, Zhang BB, Bonaldo P, Chua S, Scherer PE. Metabolic dysregulation and adipose tissue fibrosis: role of collagen VI. Mol Cell Biol. 2009;29(6):1575-91.

16. Herman MA, Peroni OD, Villoria J, Schon MR, Abumrad NA, Bluher M, Klein $\mathrm{S}$, Kahn BB. A novel ChREBP isoform in adipose tissue regulates systemic glucose metabolism. Nature. 2012;484(7394):333-8.

17. Cheng C, Haasdijk RA, Tempel D, den Dekker WK, Chrifi I, Blonden LA, van de Kamp EH, de Boer M, Burgisser PE, Noorderloos A, et al. PDGFinduced migration of vascular smooth muscle cells is inhibited by heme oxygenase-1 via VEGFR2 upregulation and subsequent assembly of inactive VEGFR2/PDGFRbeta heterodimers. Arterioscler Thromb Vasc Biol. 2012;32(5):1289-98.

18. Egashira K, Zhao Q, Kataoka C, Ohtani K, Usui M, Charo IF, Nishida K, Inoue S, Katoh M, Ichiki T, et al. Importance of monocyte chemoattractant protein-1 pathway in neointimal hyperplasia after periarterial injury in mice and monkeys. Circ Res. 2002;90(11):1167-72.

19. Komiya C, Tanaka M, Tsuchiya K, Shimazu N, Mori K, Furuke S, Miyachi Y, Shiba K, Yamaguchi S, Ikeda K, et al. Antifibrotic effect of pirfenidone in a mouse model of human nonalcoholic steatohepatitis. Scientific reports. 2017:7:44754.

20. Sun K, Tordjman J, Clement K, Scherer PE. Fibrosis and adipose tissue dysfunction. Cell Metab. 2013;18(4):470-7. 
21. Alkhouri N, Gornicka A, Berk MP, Thapaliya S, Dixon LJ, Kashyap S, Schauer PR, Feldstein AE. Adipocyte apoptosis, a link between obesity, insulin resistance, and hepatic steatosis. J Biol Chem. 2010;285(5):3428-38.

22. Boutens L, Stienstra R. Adipose tissue macrophages: going off track during obesity. Diabetologia. 2016;59(5):879-94.

23. Kalinina N, Agrotis A, Antropova Y, DiVitto G, Kanellakis P, Kostolias G, llyinskaya O, Tararak E, Bobik A. Increased expression of the DNAbinding cytokine HMGB1 in human atherosclerotic lesions: role of activated macrophages and cytokines. Arterioscler Thromb Vasc Biol. 2004;24(12):2320-5.

24. Cai J, Wen J, Bauer E, Zhong H, Yuan H, Chen AF. The role of HMGB1 in cardiovascular biology: danger signals. Antioxid Redox Signal. 2015:23(17):1351-69.

25. Shan J, Nguyen TB, Totary-Jain H, Dansky H, Marx SO, Marks AR. Leptinenhanced neointimal hyperplasia is reduced by mTOR and PI3K inhibitors. Proc Natl Acad Sci USA. 2008;105(48):19006-11.

26. Tian Z, Miyata K, Tazume H, Sakaguchi H, Kadomatsu T, Horio E, Takahashi O, Komohara Y, Araki K, Hirata Y, et al. Perivascular adipose tissue-secreted angiopoietin-like protein 2 (Angpt|2) accelerates neointimal hyperplasia after endovascular injury. J Mol Cell Cardiol. 2013;57:1-12.

27. Heldin $\mathrm{CH}$, Westermark B. Mechanism of action and in vivo role of platelet-derived growth factor. Physiol Rev. 1999;79(4):1283-316.

28. Xia M, Boini KM, Abais JM, Xu M, Zhang Y, Li PL. Endothelial NLRP3 inflammasome activation and enhanced neointima formation in mice by adipokine visfatin. Am J Pathol. 2014;184(5):1617-28.

29. Girona J, Rosales R, Plana N, Saavedra P, Masana L, Vallve JC. FABP4 induces vascular smooth muscle cell proliferation and migration through a MAPK-dependent pathway. PLoS ONE. 2013;8(11):e81914.

30. Furuhashi M, Fucho R, Gorgun CZ, Tuncman G, Cao H, Hotamisligil GS. Adipocyte/macrophage fatty acid-binding proteins contribute to metabolic deterioration through actions in both macrophages and adipocytes in mice. J Clin Invest. 2008;1 18(7):2640-50.

31. Miyachi Y, Tsuchiya K, Shiba K, Mori K, Komiya C, Ogasawara N, Ogawa Y. A reduced $\mathrm{M} 1$-like/M2-like ratio of macrophages in healthy adipose tissue expansion during SGLT2 inhibition. Sci Rep. 2018;8(1):16113.

32. Shiba K, Tsuchiya K, Komiya C, Miyachi Y, Mori K, Shimazu N, Yamaguchi S, Ogasawara N, Katoh M, Itoh M, et al. Canagliflozin, an SGLT2 inhibitor, attenuates the development of hepatocellular carcinoma in a mouse model of human NASH. Sci Rep. 2018;8(1):2362.

33. Wernstedt Asterholm I, Tao C, Morley TS, Wang QA, Delgado-Lopez F, Wang ZV, Scherer PE. Adipocyte inflammation is essential for healthy adipose tissue expansion and remodeling. Cell Metab. 2014;20(1):103-18.

34. Morley TS, Xia JY, Scherer PE. Selective enhancement of insulin sensitivity in the mature adipocyte is sufficient for systemic metabolic improvements. Nat Commun. 2015;6:7906

35. Bell CW, Jiang W, Reich CF 3rd, Pisetsky DS. The extracellular release of HMGB1 during apoptotic cell death. Am J Physiol Cell Physiol. 2006;291(6):C1318-25.

36. Li W, Sama AE, Wang H. Role of HMGB1 in cardiovascular diseases. Curr Opin Pharmacol. 2006;6(2):130-5.

37. Wang K, Li W, Yu Q, Guo B, Yang B, Zhang C, Li M, Li J, Hu S, Zheng Q, et al. High mobility group box 1 mediates interferon-gamma-induced phenotypic modulation of vascular smooth muscle cells. J Cell Biochem. 2017;118(3):518-29.

38. Kanellakis P, Agrotis A, Kyaw TS, Koulis C, Ahrens I, Mori S, Takahashi HK, Liu K, Peter K, Nishibori M, et al. High-mobility group box protein 1 neutralization reduces development of diet-induced atherosclerosis in apolipoprotein e-deficient mice. Arterioscler Thromb Vasc Biol. 2011;31(2):313-9.

39. La Cava A, Matarese G. The weight of leptin in immunity. Nat Rev Immunol. 2004;4(5):371-9.
40. Yun MR, Seo JM, Park HY. Visfatin contributes to the differentiation of monocytes into macrophages through the differential regulation of inflammatory cytokines in THP-1 cells. Cell Signal. 2014;26(4):705-15.

41. Wang P, Xu TY, Guan YF, Su DF, Fan GR, Miao CY. Perivascular adipose tissue-derived visfatin is a vascular smooth muscle cell growth factor: role of nicotinamide mononucleotide. Cardiovasc Res. 2009;81(2):370-80.

42. Furuhashi M, Fuseya T, Murata M, Hoshina K, Ishimura S, Mita T, Watanabe Y, Omori A, Matsumoto M, Sugaya T, et al. Local production of fatty acid-binding protein 4 in epicardial/perivascular fat and macrophages is linked to coronary atherosclerosis. Arterioscler Thromb Vasc Biol. 2016;36(5):825-34.

43. Fuseya T, Furuhashi M, Matsumoto M, Watanabe Y, Hoshina K, Mita T, Ishimura S, Tanaka M, Miura T. Ectopic fatty acid-binding protein 4 expression in the vascular endothelium is involved in neointima formation after vascular injury. J Am Heart Assoc. 2017;6(9):e006377.

44. Ling C, Ronn T. Epigenetics in human obesity and type 2 diabetes. Cell Metab. 2019;12(1):103.

45. Castellano-Castillo D, Moreno-Indias I, Sanchez-Alcoholado L, RamosMolina B, Alcaide-Torres J, Morcillo S, Ocana-Wilhelmi L, Tinahones F, Queipo-Ortuno MI, Cardona F. Altered adipose tissue DNA methylation status in metabolic syndrome: relationships between global DNA methylation and specific methylation at adipogenic, lipid metabolism and inflammatory candidate genes and metabolic variables. J Clin Med. 2019;8(1):87.

46. Houde AA, Legare C, Biron S, Lescelleur O, Biertho L, Marceau S, Tchernof A, Vohl MC, Hivert MF, Bouchard L. Leptin and adiponectin DNA methylation levels in adipose tissues and blood cells are associated with BMI, waist girth and LDL-cholesterol levels in severely obese men and women. BMC Med Genet. 2015;16:29.

47. Steven S, Oelze M, Hanf A, Kroller-Schon S, Kashani F, Roohani S, Welschof P, Kopp M, Godtel-Armbrust U, Xia N, et al. The SGLT2 inhibitor empagliflozin improves the primary diabetic complications in ZDF rats. Redox Biol. 2017;13:370-85.

48. GuzikTJ, Hoch NE, Brown KA, McCann LA, Rahman A, Dikalov S, Goronzy $J$, Weyand $C$, Harrison DG. Role of the T cell in the genesis of angiotensin II induced hypertension and vascular dysfunction. J Exp Med. 2007;204(10):2449-60.

49. Fitzgibbons TP, Kogan S, Aouadi M, Hendricks GM, Straubhaar J, Czech MP. Similarity of mouse perivascular and brown adipose tissues and their resistance to diet-induced inflammation. Am J Physiol Heart Circ Physiol. 2011;301(4):H1425-37.

50. Chang L, Villacorta L, Li R, Hamblin M, Xu W, Dou C, Zhang J, Wu J, Zeng R, Chen YE. Loss of perivascular adipose tissue on peroxisome proliferatoractivated receptor-gamma deletion in smooth muscle cells impairs intravascular thermoregulation and enhances atherosclerosis. Circulation. 2012;126(9):1067-78.

51. Roberts-Toler C, O'Neill BT, Cypess AM. Diet-induced obesity causes insulin resistance in mouse brown adipose tissue. Obesity (Silver Spring). 2015;23(9):1765-70.

\section{Publisher's Note}

Springer Nature remains neutral with regard to jurisdictional claims in published maps and institutional affiliations.

\footnotetext{
Ready to submit your research? Choose BMC and benefit from:

- fast, convenient online submission

- thorough peer review by experienced researchers in your field

- rapid publication on acceptance

- support for research data, including large and complex data types

- gold Open Access which fosters wider collaboration and increased citations

- maximum visibility for your research: over 100M website views per year
}

At BMC, research is always in progress.

Learn more biomedcentral.com/submissions 